\title{
First Few Cases of Intracranial Schwannoma treated with LINAC-based Stereotactic Radiosurgery in Bangladesh
}

\author{
Narendra Kumar' ${ }^{1}$ Taohida Yasmin², Murugan Appasamy³, Sania Ahsan, KM Masud Rana \\ Sandip Kumar Das ${ }^{6}$, R Arun Kumar ${ }^{7}, Z_{1}$ illur Rahman ${ }^{8}$, Amitabha Chanda $^{9}$, Md. Shafiul Alam ${ }^{10}$ \\ ${ }^{1}$ Professor \& Head, Department of Radiation Oncology, Cancer Care Centre, Evercare Hospital, Dhaka, Bangladesh; ${ }^{2}$ Registrar, Department \\ of Radiation Oncology, Cancer Care Centre, Evercare Hospital, Dhaka, Bangladesh; ${ }^{3}$ Chief Medical Physicist, Department of Radiation \\ Oncology, Cancer Care Centre, Evercare Hospital, Dhaka, Bangladesh; ${ }^{4}$ Senior Consultant, Department of Radiodiagnosis, Evercare \\ Hospital Dhaka, Bangladesh; ${ }^{5}$ Medical Physicist, Department of Radiation Oncology, Cancer Care Centre, Evercare Hospital, \\ Dhaka, Bangladesh; ${ }^{6}$ Senior Consultant, Department of Neurology, Evercare Hospital Dhaka, Bangladesh; ${ }^{7}$ Chief Radiation \\ Technologist, Department of Radiation Oncology, Cancer Care Centre, Evercare Hospital, Dhaka, Bangladesh; ${ }^{8}$ Senior \\ Consultant, Department of Neurosurgery, Evercare Hospital Dhaka, Bangladesh; ${ }^{9}$ Senior Consultant, Department of \\ Neurosurgery, Evercare Hospital Dhaka, Bangladesh; ${ }^{10}$ Associate Professor, Department of \\ Gamma Knife, National Institute of Neurosciences \& Hospital, Dhaka, Bangladesh
}

[Received on: 22 April 2021; Accepted on: 12 May 2021; Published: 1 July 2021]

\begin{abstract}
Stereotactic Radiosurgery has been widely utilized for the treatment of intracranial lesions, and this paper presents our experience in treating schwannomas in Bangladesh. This case series was described to present our institutional experience and procedural technique adapted for treating intracranial schwannomas using stereotactic Radiosurgery (SRS) by Linear accelerators. This case series since starting our radiosurgery program in March 2019, we have treated three patients of intracranial schwannoma. Two patients had vestibular schwannoma, and one had Trigeminal Schwannoma. All three patients first underwent surgical intervention and on recurrence/progression treated with stereotactic Radiosurgery. Tumor volume ranged from 4.47 to $10.22 \mathrm{~cm} 3$. The dose prescription ranges from 13 to $14 \mathrm{~Gy}$ in one or two fractions, which was subject to tumor volume, its proximity to a critical structure, existing neurologic deficit, and optimal balance between prescribed dose and predicted complications. All three procedure was free of any immediate adverse event. LINAC based Stereotactic Radiosurgery was found to be a safe and effective option for the treatment of schwannomas. All three patients have a very good clinical outcome, and one patient who has more than one-year post-SRS revealed a significant regression in the size of the tumor. [Journal of National Institute of Neurosciences Bangladesh, July 2021;7(2):181-189]
\end{abstract}

Keywords: LINAC; Stereotactic Radiosurgery; trigeminal schwannoma; vestibular schwannoma; complications

Correspondence: Prof. Dr. Narendra Kumar, Professor \& Head, Department of Radiation Oncology, Cancer care Centre, Evercare Hospital, Basundhora Residential Area, Dhaka-1229, Bangladesh; Cell no.: +8801777763535; Email: narendra.kumar@evercarebd.com; drnarendra74@gmail.com

Conflict of interest: There is no financial conflict of interest relevant to this paper to disclose.

Funding agency: This research project was not funded by any group or any institution.

Contribution to authors: Kumar N, Yasmin T, Appasamy M, Ahsan S, Rana KMM have contributed from the protocol preparation, data collection up to report writing. Manuscript writing has been performed by Kumar N. However, Das SK, Kumar RA, Rahman Z, Chanda A, Alam MS have revised the manuscript.

How to cite this article: Kumar N, Yasmin T, Appasamy M, Ahsan S, Rana KMM, Das SK, Kumar RA, Rahman Z, Chanda A, Alam MS. First Few Cases of Intracranial Schwannoma treated with LINAC-based Stereotactic Radiosurgery (SRS) in Bangladesh. J Natl Inst Neurosci Bangladesh, 2021;7(2): 181-189

Copyright: (C2021. Kumar et al. Published by Journal of National Institute of Neurosciences Bangladesh. This article is published under the Creative Commons CC BY-NC License (https://creativecommons.org/licenses/by-nc/4.0/). This license permits use, distribution and reproduction in any medium, provided the original work is properly cited, and is not used for commercial purposes.

\section{Introduction}

Intracranial Schwannomas or Neurilemmomas are benign and mostly slow-growing tumors. It originates from the nerve sheath cell (Schwan cell) covering the nerves and comprises $5-9 \%$ of all intracranial tumors. In which, approximately $90 \%$ of intracranial schwannomas arise from eighth cranial nerve termed as vestibular schwannomas (VSs) or Acoustic neuroma ${ }^{1}$. Non-acoustic 
schwannomas account for $10 \%$ of all intracranial schwannomas \& can contain any of Cranial Nerve V, VII, IX, X, or XI. Trigeminal schwannomas (TS) are the second most common cranial nerve schwannomas though it accounts for $0.8-8 \%$ of all intracranial schwannomas, and only $0.07-0.28 \%$ of all intracranial tumors ${ }^{2}$. The median age at diagnosis for VSs is 55 years. $90 \%$ of the tumors are unilateral at presentation. Bilateral VSs are commonly linked to type 2 Neurofibromatosis $(\mathrm{NF} 2)^{3}$. Trigeminal and facial neuropathies, vertigo, and ataxia are the most common physical symptoms. In a larger tumor obstructive feature, brainstem compression, dilated ventricle, hydrocephalus, or hearing loss are more noticeable. TSs are seen in all age groups and can arise from trigeminal nerve anywhere of its cranial and extracranial portion. Facial pain, paresthesia, and masticator muscle weakness are the most common symptoms due to trigeminal and its adjacent cranial nerve dysfunction. A subgroup of the patient remains asymptomatic and diagnosed incidentally only ${ }^{4}$.

Management options of intracranial Vestibular schwannomas include observation with serial imaging, surgical excision and stereotactic radiosurgery (SRS), and fractionated stereotactic radiotherapy (FSRT). An asymptomatic old patient with a small tumor in the absence of cranial nerve palsy is advocated for observation. In general prompt intervention helps to halt the tumor growth and prevent progression of neural deficit $^{5,6}$. Incidentally found trigeminal schwannomas of any age can be managed conservatively with imaging series. Traditionally, Complete Surgical resection is the orthodox treatment for all intracranial Schwanomma. However, peri or post-operative new neurological deficit still a concern even in the era of the modern surgical approach ${ }^{7}$. In this regard, stereotactic radiosurgery (SRS) has been a successful substitute for controlling tumor and preserving Neurological function ${ }^{8}$. In addition to that, SRS has been a preferred treatment option for medically inoperable patients, patients in whom significant morbidity is expected due to an extensive surgical approach or patients with incomplete resection". Stereotactic radiosurgery is an advanced technique of external beam radiation therapy where multiple high dose rate radiation beams are precisely directed to the target lesion in such a fashion that high dose radiation is tightly conformed to produce desired radiobiological response and to minimize radiation dose to surrounding normal structure by creating sharp dose fall-off outside the target. SRS exploits the normal cellular mechanism and radiobiology of tumor cells by causing shrinkage of the tumor by its cytotoxic effect and obliteration of blood vessels due to endothelial injury and mural hyalinization, hemosiderin deposits and luminal closure ${ }^{10}$. There are abundant radiosurgery series supporting the distinct role Gamma Knife Radio Surgery (GKRS) in the management of intracranial schwannomas ${ }^{11,12}$. GKRS unit Consist of $201 \mathrm{Co}^{60}$ Sources arranged in sphere-shaped hamlet with a beam diameter of 1 to $4 \mathrm{~mm}$ to precisely deliver the desired dose to a smaller target. Development in Linear Accelerator made the Linac-based Stereotactic Radiosurgery as an alternative where dedicated GKRS facilities are not available. A LINAC unit, non-invasive stereotactic head frame, modern treatment planning systems along with comprehensive QA test are the component of a Lina-Based SRS system and has the capacity to generate superior dose-effect than GKRS with maximum dose to the tumor with higher Conformity Index (CI) and minimum dose to the critical structure with higher Gradient Index (GI).

In this case series, we intend to share our institutional experience in treating three patients with intracranial schwannoma, who underwent Linac Based Stereotactic Radiosurgery in our institution in the last one year.

\section{Case Presentation 1}

A 57-year-old man, known diabetic \& hypertensive, in October 2015, developed pain with an occasional tingling sensation on the left side of face and eye for two months. The pain used to persist for 5-10 mins and frequency of 10-15times per day. On physical examination, he was found to have left-sided facial hyperalgesia and mild ptosis of the left eye. Brain MRI Suggested left trigeminal schwannoma. He started with Gabapentin, Amitriptyline, and Mecobalamin for Neuralgic Pain. Later in Dec'2015, he underwent Gamma Knife Radiosurgery (GKRS) with $12 \mathrm{~Gy}$ in India. His pain found to reduce in two months \& was complain free. Lost followup In March'2019, almost 51-month post-GKRS, he presented to the Radiation Oncology department of Evercare hospital Dhaka with the complaint of pain and numbness over the left side of the face for three months. MRI showed $-3.8 \times 2.6 \mathrm{~cm}$ left trigeminal nerve, mass effect on the left side of Pons, and left middle cerebellar peduncle. $\mathrm{He}$ had no new significant neurologic deficit. As the tumor size was smaller with no major cystic component inside the tumor, options of repeat radiosurgery and microsurgery were explained in detail to the patient. Having more than four years of gap between initial GKRS and relapse, the role of repeat radiosurgery discussed in detail with the patient and a consensus decision was made to treat with 
multisession Stereotactic Radiosurgery. He was treated with a dose of 14Gy in two fractions treated on 21.03.19 and 22.03.19. Three months after SRS, there was more than $90 \%$ improvement in the pain, but mild numbness over the left side of the face was still present. Follow-up assessment at six months in October 2019, there was a complete disappearance of pain with no neurological deficit, and Radiological assessment with MRI was suggestive of stable disease. His next follow up was scheduled in April 2020. We came to know that patient passed away in December 2019 due to high-grade fever and subsequent effects on telephonic inquiry.

\section{Case Presentation 2}

Our second case was a 48-year-old lady, who was presented in the Department of Neurology in Dec'2015, with the complains of decreased sensation over the left side of the face for six months, headache, sleep disturbance, weakness of left side of face \& imbalance in walking and difficulty in hearing in the left ear. Physical examination revealed wasting of Temporalis muscle with cranial nerve deficit of left V(Both), VII, VIII with cerebellar palsy. MRI of the brain showed -Left CP angle tumor of $3.5 \mathrm{~cm}$ size without hydrocephalus; Left Trigeminal Schwannoma. She underwent posterior fossa craniotomy and excision of the tumor. Histopathology came out to be schwannoma. After 4-week post-surgery, she had no neurological deficit and started on gabapentin for headache and advised to review after three months. In May'16, she developed mild left facial palsy, and CECT revealed a residual left $\mathrm{CP}$ angle tumor of $2.9 \times 1.3 \mathrm{~cm}$ size, mildly compressing the left side of the Pons and left middle cerebellar peduncle. She was continued on medical management. In Jun 2017 (after one year) on routine follow up assessment patient had hypoesthesia over the left side of face; however, radiological assessment with MRI showed the stable residual tumor in $\mathrm{CP}$ angle of $2.6 \times 2.6 \mathrm{~cm}$ size, mild mass effect on the left side of the Pons. The patient did not turn up for 20 months. In March'19, she presented with the complaint of electric shock-like pain all over the left face. MRI was suggestive significantly increase the tumor with increased mass effect on left Pons and Middle cerebellar peduncles. The patient was planned for Stereotactic Radiosurgery. She received stereotactic Radiosurgery with dose of 13Gy in two fractions treated on 15.04.2019 and 16.04.2019. Two months after SRS, there was a significant clinical improvement with more than $90 \%$ improvement in the pain. MRI at four months of SRS showed little increase in tumor size of $3.2 \times 2.6 \times 2.9 \mathrm{~cm}$ with mild surroundings edema; however, she was clinically asymptomatic. Considering it as radiation-induced, she was prescribed low dose steroids for one month and advised the next follow up after six months. On her last follow up in Jun 2020, 14-months post-SRS, she was clinically asymptomatic without any neurological deficit. MRI revealed an impressive regression of tumor very well as expected after year post-SRS in case of schwannoma. The current tumor volume is $4.76 \mathrm{cc}$ compared to initial tumor volume (GTV) of $7.89 \mathrm{CC}$, an approximately $38 \%$ reduction in tumor volume observed.

\section{Case Presentation 3}

The third case was a 61-year old gentleman, known hypertensive. He was presented in the department of neurosurgery in August 2019 with the complaint of severe pain in the right eye, jaw, and tingling sensation over the right side of the face for one month. He first time noticed pain over his right side of the face about 15 years back, which was very infrequent and of very short duration and get relieved by taking some analgesic. Now he had severe pain with longer duration and more frequent. MRI brain revealed an extra-axial soft tissue mass of $2.7 \times 1.7 \mathrm{~cm}$ size in Right $\mathrm{CP}$ angel involving the root entry zone of right $7^{\text {th }}$ and $8^{\text {th }}$ cranial nerve. He was diagnosed as a case of right Vestibular Neuroma. On clinical evaluation, there was no neurological deficit. The patient had been discussed in detail about the treatment option of surgery and Stereotactic Radiosurgery. He was very much suitable for Stereotactic Radiosurgery. The patient had been discussed in detail about the role of SRS both in terms of outcome and possible acute and late side effects. He received a stereotactic Radiosurgery dose of 13Gy on 28.09.2019. On his first follow-up after three months in December 2019, He was clinically stable without any complaints. The patient was planned six months post-SRS radiological assessment in April 2020, but due to the current Covid-19 pandemic, he could not come for MRI.

In 2019, 3 patients of benign intracranial schwannoma were treated with Stereotactic Radiosurgery at our hospital. Two were male $\&$ one female. The tumor was located on the left side in two patients and on Right in one patient. Two patients had prior intervention (resection/SRS). Radiosurgery was given for recurrent or progressive or symptomatic tumors. Two patients had schwannoma of the trigeminal nerve, and one patient had vestibular schwannoma. Tumor volume ranged from 4.47 to $10.22 \mathrm{~cm} 3$. Out of these three patients, one patient treated with primary Stereotactic Radiosurgery alone, one patient received SRS after the progression of tumor 
Table 1: Patients demographic and previous treatment characteristic

\begin{tabular}{|c|c|c|c|c|c|c|}
\hline Patient no & Age/sex & Side & Tumor location & Symptoms & Pre-SRS treatments & Tumor volume PTV $(\mathrm{cm} 3)$ \\
\hline 1 & $57 / \mathrm{M}$ & $\mathrm{L}$ & Trigeminal nerve & Trigeminal neuralgia \& & GKRS x1 & 13.8 \\
\hline 2 & $48 / \mathrm{F}$ & $\mathrm{L}$ & & Trigeminal paraesthesia & & \\
\hline \multirow[t]{2}{*}{3} & $61 / \mathrm{M}$ & $\mathrm{R}$ & Trigeminal nerve & $\begin{array}{l}\text { trigeminal neuralgic } \\
\text { pain }\end{array}$ & Surgical excision $\mathrm{x} 1$ & 11.2 \\
\hline & & & $\begin{array}{c}\text { Facial and } \\
\text { Vestibulocochlear } \\
\text { nerve }\end{array}$ & $\begin{array}{l}\text { Facial pain \& } \\
\text { paresthesia }\end{array}$ & None & 6.6 \\
\hline
\end{tabular}

post-surgery, and one patient received repeat Stereotactic Radiosurgery for the relapse of the tumor after five years from first GKRS treatment. A complete history, physical examination, review of previous medical records were made before instigation of treatment. Facial pain was the most common presenting symptom in all three patients. Table 1 summarizes the demographic profile and treatment characteristics of each patient.

Radiotherapy Techniques \&Treatments: All the patients treated on the Linac-based stereotactic system at Evercare Hospital Dhaka. They have been referred to our department for treatment with recurrent and symptomatic diseases after surgery, GKRS, and patients with tumors in medically inoperable or eloquent areas. After consultation with Radiation Oncologist, the patient is decided for stereotactic Radiotherapy, informed written consent is obtained from patient \& family members. Treatment decisions for all patients were based on a comprehensive assessment of tumor size, patient complaints and performance status, cranial nerve functions, treatment history, and personal preference. During the Stereotactic radiosurgery treatment process, patients were positioned in the supine position on special head support (Elekta Fraxion) were immobilized using a double layer thermoplastic mask with three clamps. A " $Z$ " shaped non-invasive localizer box is also used during the setup process that gives nine external co-ordinates or localizing marker on the axial slice. This external localizer box helps us in locating the isocenter of the treatment plan through stereotactic co-ordinates in locating the tumor to the accuracy of the submillimeter during Stereotactic treatment delivery.

After mold work is complete, the patient is taken to the CT scanner (GE, Discover VCT) for contrast-enhanced CT-scan simulation. Images are obtained at $1 \mathrm{~mm}$ thickness in axial images of with stereotactic localizer box in situ. Afterward, a high-resolution Gadolinium-enhanced T1 weighted MRI of similar thickness was acquired in the same setup (Figure I). Both CT-scan and MRI were co-registered in the treatment planning system. Radiation Oncologist, Neurosurgeon, and Neuroradiologist delineated the target and the Organ at Risk (OARs) as the volume of interest on Gadolinium-enhanced MRI. OAR's included brainstem, temporal lobe, cochlea (Figure II).
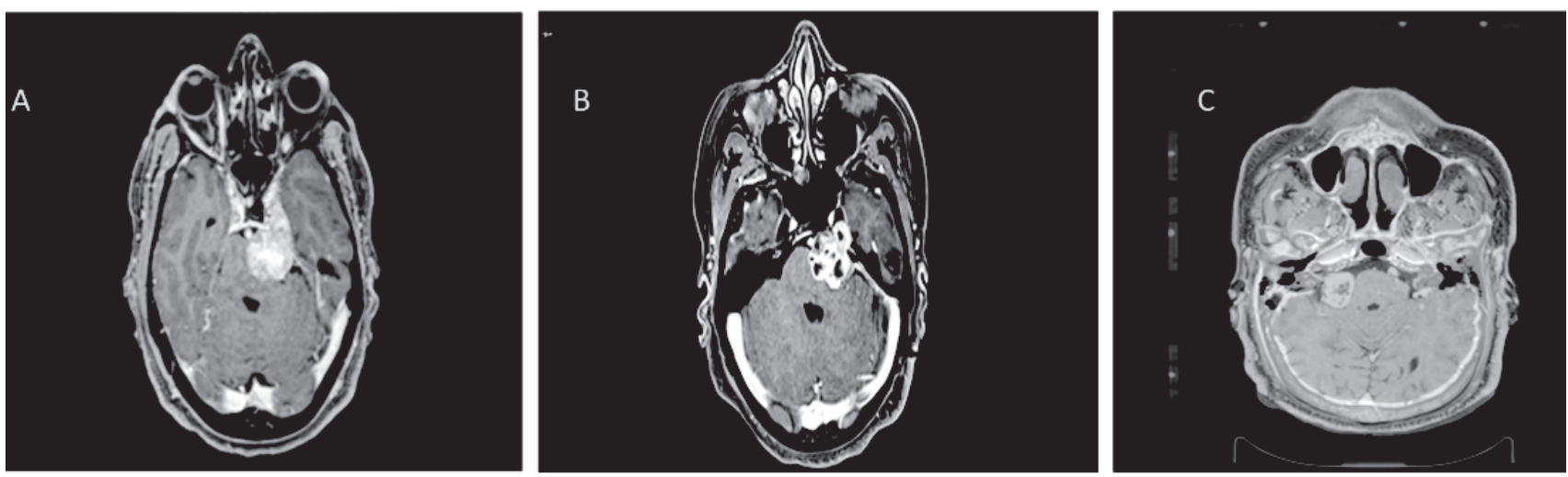

Figure I: T1 weighted MRI image showing contrast-enhanced extra-axial soft tissue mass. (A) patient 1, 57yr-male with left trigeminal schwannoma (TS), previous GKRS, had repeat SRS after 51months due to progression (B) patient 2, 48yr-female with left trigeminal schwannoma (TS), Previous excision, had SRS after 38 months due to progression (C) patient 3, 61yr-male with right vestibular neuroma with a 15-year history of slow tumor progression underwent radical SRS. 

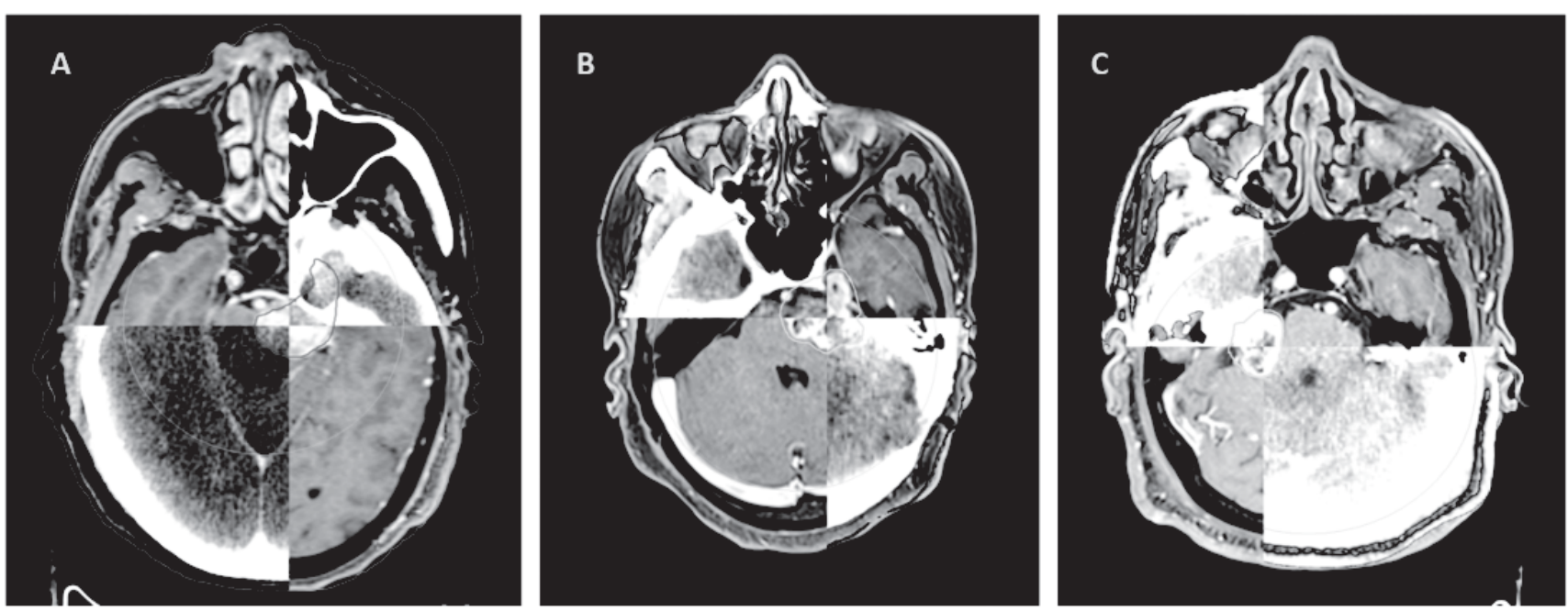

Figure II: Showing fusion (Co-Registration) of MRI images with CT-scan for delineation of target followed by planning. (A) patient 1, (B) patient 2 and (C) patient 3.
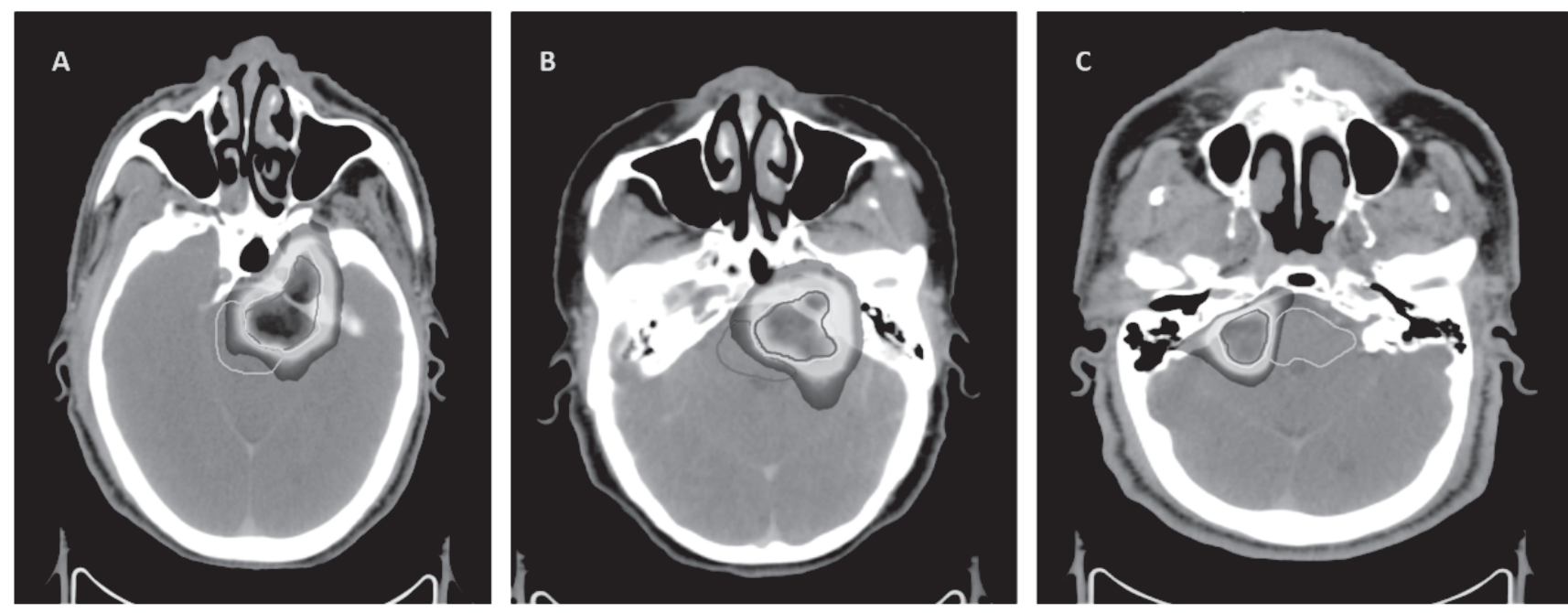

Figure III: The dose distribution achieved by the Monaco Treatment Planning System (Elekta, Version 5.3). (A) patient 1 (B) patient 2 and (C) patient 3.

When the prescription dose was selected, Treatment planning was performed by medical physicists using the Monaco treatment planning system (Elekta, Version 5.3). We have used $3-4$ non-coplanar irregular shaped partial arcs for SRS treatment in the VMAT inverse treatment technique (Figure III). The aim was to achieve the maximum dose in the target volume and minimum dose to surrounding critical structures.

Treatment plans were reviewed vigilantly by Treating Radiation Oncologist and Medical Physicist against

Table 2: Patients Dosimetric Characteristics

\begin{tabular}{|c|c|c|c|c|c|c|c|c|c|c|c|}
\hline $\begin{array}{l}\text { S. } \\
\text { N. }\end{array}$ & $\begin{array}{l}\text { Dose (Gy)/ } \\
\text { No. of } \\
\text { Fractions }\end{array}$ & $\begin{array}{c}\text { PTV } \\
D_{\max }(G y)\end{array}$ & $\begin{array}{l}\text { PTVD } 95 \\
\text { (Gy)* }\end{array}$ & $\begin{array}{l}\text { PTVD99 } \\
(\text { Gy)*** }\end{array}$ & HI\# & CI\#\# & GI\#\# & $\begin{array}{l}\text { Hot } \\
\text { spot }\end{array}$ & $\begin{array}{l}\text { Brain stem } \\
\text { maximum dose } \\
\text { (Gy) }\end{array}$ & $\begin{array}{c}\text { Ipsilateral } \\
\text { Cochlea mean } \\
\text { dose (Gy) }\end{array}$ & $\begin{array}{l}\text { Optic Chiasma } \\
\text { mean dose } \\
(\mathrm{Gy})\end{array}$ \\
\hline 1 & $14 / 2$ & 21.28 & 14.8 & 13.8 & 1.38 & 0.81 & 0.23 & $152 \%$ & 15.07 & 6.4 & 2.1 \\
\hline 2 & $14 / 2$ & 17.39 & 12.19 & 11.07 & 1.37 & 0.62 & 0.22 & $139 \%$ & 12.2 & 6.4 & 1.3 \\
\hline 3 & $13 / 1$ & 17.85 & 12.54 & 11.14 & 1.35 & 0.80 & 0.27 & $137 \%$ & 8.48 & 1.65 & 4.6 \\
\hline
\end{tabular}

*PTVD95 - Dose Covered by 99\% of PTV, **PTVD99 - Dose covered by 99\% of PTV; \#HI-Heterogeneity Index, \#\#CI-Conformity Index, \#\#\#G-Dose Gradient Index 

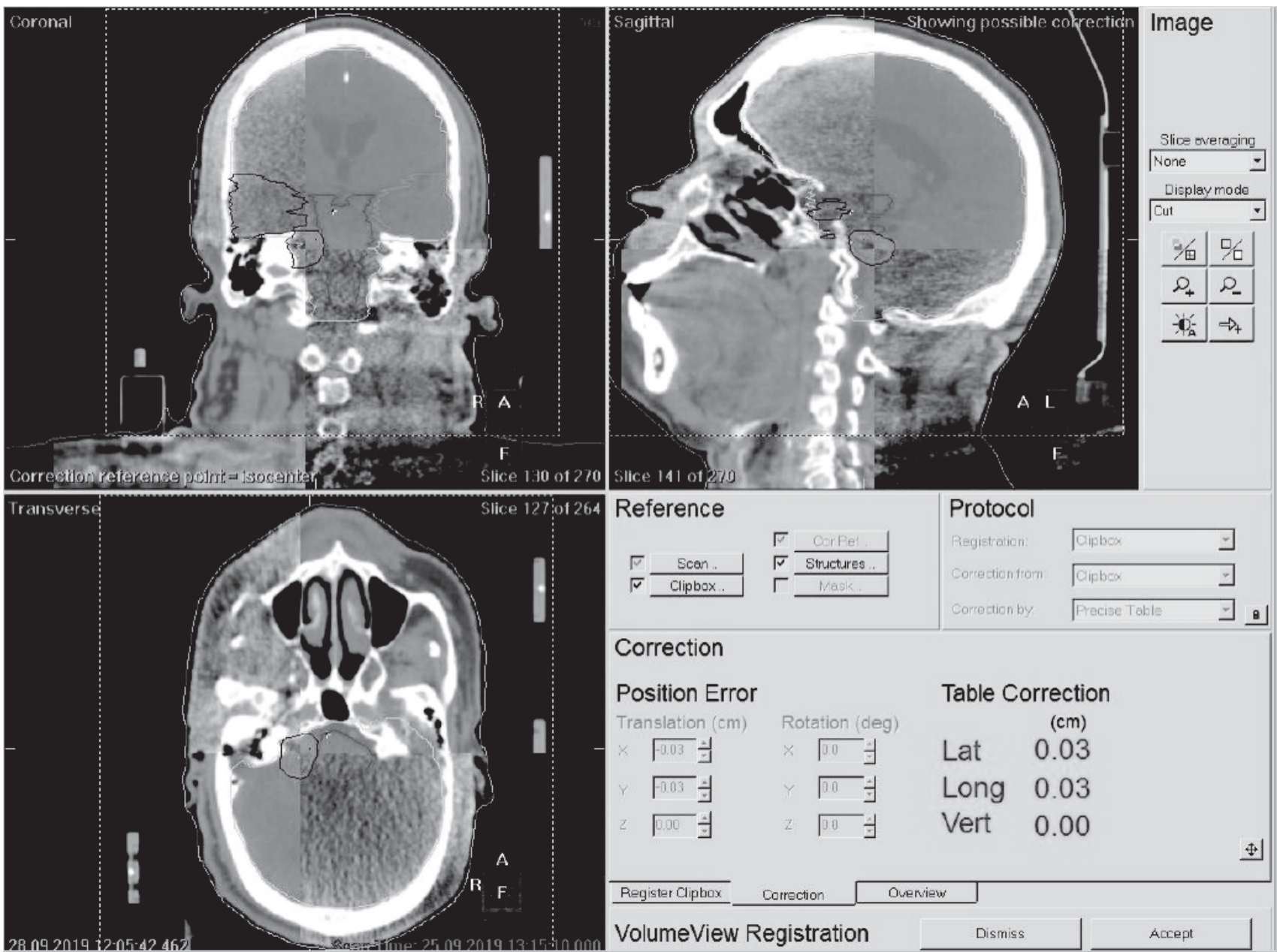

Figure IV: shows the kVCT verification for the third patient, co-registered with planning CT image, and obtained table correction prior to treatment to assure the patient positioning in sub-millimeter accuracy

The Radiation Therapy Oncology Group (RTOG) dose constraints, Conformity index and Homogeneity index, and Dose Gradient Index. System generated dose-volume histogram (DVH) is analyzed before final approval. And keen attention was paid for the hot and cold spots inside and outside the target volume before the plan being approved for treatment. The table below illustrates the quantitative and qualitative dosimetric characteristics of all three patients recommended dose coverage for the target, and OAR's has been strictly maintained.

A patient-specific QA was done after the plan is approved. This QA process ensures the delivery of the intended dose within the tolerance limit, and a dry run was carried out to ensure collision-free treatment.

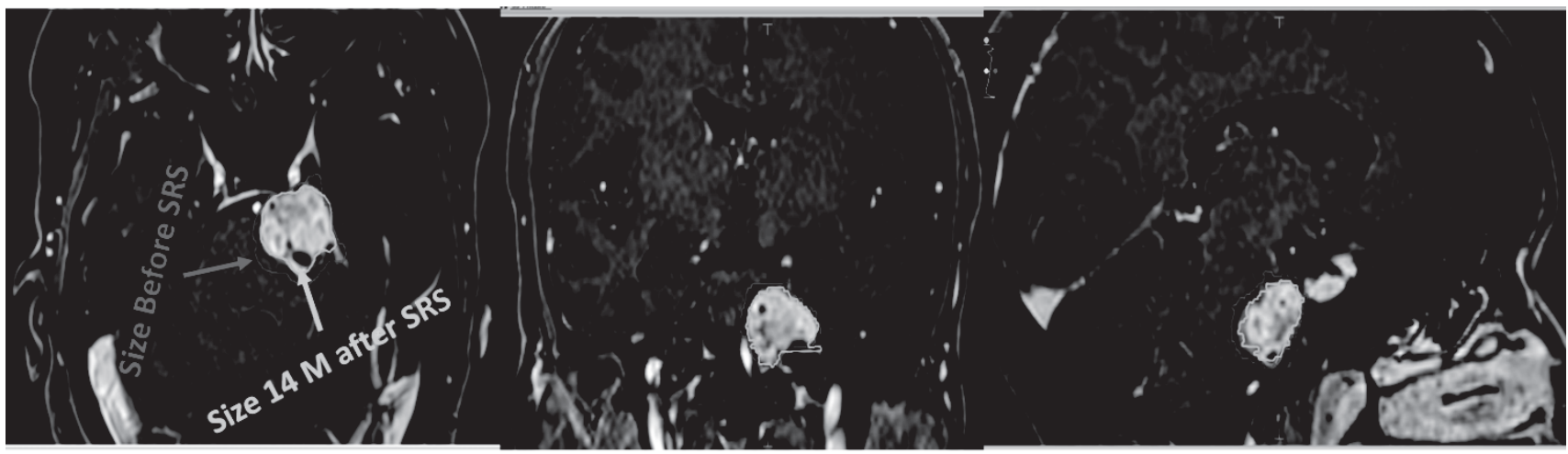

Figure V: Follow-up MRI scan shows patient Number 2, showing the tumor regression after 14 months of SRS 
Before treatment, reproducibility of the patient position was verified using KVCT \& hexapod 6D couch prior to radiation delivery to ensure submillimeter accuracy (Figure IV).

The Tumor dose was ranged from 12-14 Gy. The prescribed dose was given either in a single session or in two sessions considering the tumor volume, location of critical normal organs, existing neurologic deficit, and anticipated complications. For multisession treatment, a 24-hour time interval was maintained. All SRS patients were given prophylactic steroid, antiemetic \& mannitol on the day of SRS treatments to reduce the risk of acute edema. Steroids \& antiemetics were tapered off over two to three weeks.

Toxicity Evaluation: Common Terminology Criteria for Adverse Events version 5.0 (CTCAE) has been used. Acute toxicities were defined as toxicities observed within three months of radiation therapy. CTCAE Grade-1 fatigue and ataxia was present in one patient. Complications \& Clinical Response: All the patients were free of any adverse event in a 24hour post-procedural period. Initial Follow up was done after 2 to 4 months of radiation therapy. All the patients were free of treatment-related complications. They had improvement in pain and headache, which was the most common complaint among all the patients. No new cranial deficit was observed.

Follow up Evaluation: Follow-up included periodic review including neuroradiological evaluation, comprehensive and history taking, and physical examination, including nerve function assessment. Two patients who underwent MRI at 4 and 7-months post-SRS, one showed a mild increase in size, and second, have a stable tumor. Radiobiological behavior suggests for an appropriate radiological assessment 2 to 3 years follow up is needed. One patient who has undergone an MRI after 14 months showed an impressive regression in the tumor volume. However, the purpose of this case series reporting is mainly to present symptomatic improvement as well as SRS treatment-related complications (Figure 5).

\section{Discussion}

Intracranial schwannomas are non-malignant tumors that arise from the covering nerve sheath. It is less common and encompasses about $5.0 \%$ to $9.0 \%$ of the intracranial tumor. Vestibular schwannoma and trigeminal schwannoma are the two most common intracranial schwannomas. This case series report discusses the management of three patients with such conditions.

Management of Intracranial schwannomas includes observation, surgery, SRS, FSRT, or in combination. Initially, SRS was done using the GKRS unit. Subsequently, LINAC based SRS has also upheld its domain of being able to deliver a higher dose to the target lesion with more précised dose delivery and sharper dose fall of outside the PTV. Improvement in imaging and treatment planning techniques has contributed to more promising local controls and reduced complications. Table 3 below displays a few studies of SRS for intracranial schwannoma. It is found that outcome of SRS in terms of Local control \& morbidity is fairly similar in GKRS unit \& Linac Based SRS system.

Observation of Intracranial Schwannoma is limited for the elderly patients who are having a very small-sized or incidentally detected tumor, of which the growth rate is expected to be low with no significant clinical symptoms, neurological deficit, or pressure effects. This "watch-and-wait" strategy is also dependent on the patient's reliability to attend regular follow up. Patients are monitored with periodic MRI and cranial nerve

Table 3: few Literatures Summery using SRS in Intracranial Schwannoma

\begin{tabular}{lccccccc}
\hline Reference & $\begin{array}{c}\text { Patients } \\
\text { (n) }\end{array}$ & Diagnosis & Modality & $\begin{array}{c}\text { Mean } \\
\text { dose }\end{array}$ & $\begin{array}{c}\text { Median } \\
\text { follow-up (years) }\end{array}$ & $\begin{array}{c}\text { Local } \\
\text { control }\end{array}$ & $\begin{array}{c}\text { Morbidity/ new } \\
\text { facial neuropathy }\end{array}$ \\
\hline Combs $^{16}$ & 26 & acoustic neuroma & Linac & $13 \mathrm{~Gy}$ & 9 & $91.1 \%$ & $5 \%$ \\
Hayhurst $^{17}$ & 73 & Vestibular schwannoma & GKRS & $12 \mathrm{~Gy}$ & 2.4 & $96 \%$ & $3.7 \%$ \\
Murphy $^{18}$ & 103 & Vestibular schwannoma & GKRS & $13 \mathrm{~Gy}$ & 5 & $91.1 \%$ & $5 \%$ \\
Benghiat $^{19}$ & 97 & Vestibular schwannoma & Linac & $12 \mathrm{~Gy}$ & 2.4 & $100 \%$ & $2.1 \%$ \\
Mebanta $^{20}$ & 18 & Non-acoustic schwannoma & Linac & 13.1 & 2.8 & $100 \%$ & $17 \%$ \\
Pan et al $^{21}$ & 56 & Trigeminal schwannoma & GKS & 13.3 & 5.6 & $93 \%$ & 7.1 \\
Nettel et al $^{22}$ & 23 & Trigeminal schwannoma & GKS & 15 & 3.3 & $91 \%$ & $8.6 \%$ \\
Peker $^{2}$ & 45 & Trigeminal schwannoma & GKS & 16 & 5 & $100 \%$ & $6.7 \%$ \\
\hline
\end{tabular}

*PTVD95 - Dose Covered by 99\% of PTV, **PTVD99 - Dose covered by 99\% of PTV; \#HI-Heterogeneity Index, \#\#CI-Conformity Index, \#\#\#GI-Dose Gradient Index 
function assessment. Published data also suggests that early active intervention versus watchful observation should be balanced with the judicial assessment of specialists concerning symptomatic progression, cranial nerve dysfunction, and often losing treatment options while waiting ${ }^{13,14}$. In our case series, one of the patients with Right vestibular schwannoma had a prolonged history of 15 years. He did not seek medical attention unless his symptoms were hindering his normal day-to-day life. MR scan in Aug'19 suggested a small tumor of $27 \times 17 \mathrm{~mm}$. His tumor probably had a slow growth rate. As he was symptomatic afterward, the active intervention has been recommended.

The Role of Radiosurgery in Cranial Schwannoma has been established as a primary or adjuvant treatment in case of subtotal resection or tumor progression after complete resection or cases where re-resection is not possible ${ }^{23,26,27}$. As a matter of fact, SRS offers a better alternative of surgery for medically infirm cases in respect of Peri \& Post-operative morbidity, including cranial neuropathy and CSF leakage15. In our case series, one patient has tumor progression 38 months after excision who received SRS.

In our case series, one patient had repeat Radiosurgery for trigeminal schwannoma, although there is a lack of sufficient literature supporting repeat SRS for trigeminal schwannoma. A review article by MacNally et al. mentioned about progression after Radiosurgery was treated selectively by Repeat Radiosurgery9. In other Studies of vestibular schwannoma, patients having tumor progression or recurrence after 03 years of GKRS, small tumor volume, no new significant neurological deficit, no sign of cerebellar ataxia, or pyramidal tract was the indication of repeat Radiosurgery ${ }^{24}$. Our patient had tumor progression 51 months, followed by Primary GKRS. He had a small-sized tumor $(3.8 \times 2.6 \mathrm{~cm})$ with no new neurological deficit.

In this series, two of the patients received multisession stereotactic Radiosurgery for trigeminal schwannoma. Although the event of SRS implicates prescribed doses to be delivered over a single session as OPD procedure has excellent tumor control as illustrated in the table, the risk of treatment-related cranial nerve palsy or dysfunction has been a major concern. To improve functional outcome without compromising tumor control, dose de-escalation of the prescribed dose (from 16-20 Gy to 12-13Gy) and a number of sessions has been investigated24,26. The Radiobiological principles of fractionation also suggest less toxicity of normal tissue by allowing sublethal normal tissue damage repair in multiple fractions ${ }^{25}$. Hansasuta et al. published a single institution experience of 383 cases. They treated patients with a dose of $18 \mathrm{~Gy}$ in 3 sessions for tumor volume of 0.02 to $19.8 \mathrm{~cm}^{3}$. They reinforced a superior hearing preservation rate and less non-auditory complications in multisession SRS. Authors also motioned about younger age and smaller tumor volume as a positive prognostic inde ${ }^{26}$. In our series, as the tumor was smaller in size, they were prescribed 14Gy radiation in 2 sessions.

Among all three patients, two patients could do their first MRI at $5 \& 7$ months post-SRT to assess tumor size. One of which had a mild increase in size, and the other was stable. Although it is very difficult to comment on this short interval imaging, the literature suggests $<29 \%$ of tumors may have a transient increase in volume after $\mathrm{SRS}^{28}$. One patient whose MRI at five months indicates little increase in size but at 14 months clearly showed a regression in the size of the tumor. Patients are required to follow up at least 3-5 years to comment on local control. Among the three patients, one patient follow-up on $14^{\text {th }}$ month showed a tumor regression of about $38 \%$ compared with that of baseline. Published Data on LINAC based Radiosurgery for vestibular and non-acoustic schwannoma has local control ranging from $84-100 \%{ }^{19-20,28}$.

All three patients had a significant reduction in the symptoms with no notable Radio surgical complications. One patient had CTCAE Grade-1 fatigue and preexisting ataxia. Data suggests treatment-related toxicity includes worsening of pre-existing nerve palsy, new onset of hearing loss, ataxia, transient facial/trigeminal neuropathy, hemifacial spasm, very rarely obstructive hydrocephalus, necrosis within the temporal lobe ${ }^{19-20}$.

The advantage of LINAC based SRS is it does not require an invasive stereotactic frame that is needed to be fixed over patients' skull. That allows more patient compliance. In LINAC based SRS prescribed dose can be divided into multiple sessions when indicated. With the advent of the modern planning system, the purpose of achieving rapid dose fall-off outside the PTV can be gained by using a multi-leaf collimator, prioritized dose falls off to organ at risks, and improved conformity index (CI) \& Homogeneity Index (HI). SRS can be done as an outpatient basis. In our series, we have been able to attain better conformality for three patients.

\section{Conclusion}

LINAC-base SRS is a suitable and very effective option of treatment for intracranial schwannomas. It is found to be more convenient, having low treatment-related complications. A longer follow-up 
period of 2-3 years is needed for appropriate radiological assessment of the tumor and late side effects.

Acknowledgment: The authors would like to express their sincere thanks to the Management of Evercare Hospitals Dhaka for providing the facility and continuous support. They also acknowledge Radiology and Neurology and Neurosurgery Departments for their continuous support.

\section{References}

1. Zabel A, Debus J, Thilmann C, Schlegel W, Wannenmacher M. Management of Benign cranial non-acoustic schwannomas by fractionated stereotactic Radiotherapy. International journal of cancer. 2001;96(6):356-362

2. Peker S, Bayrakli F, Kilic T, Pamir MN. Gamma-knife radiosurgery in treatment of trigeminal schwannoma. Acta Neurochirurgica. 2007; 149:1133-1137

3. Lanser MJ, Sussman SA, Frazer K. Epidemiology, pathogenesis and genetics of acoustic tumors. Otolaryngologic Clinics of North America. 1992; 25:499-520

4. Kondziolka D, Shin SM, Brunswick A, Kim I, Silverman JS. The biology of Radiosurgery and its clinical applications for brain tumors. Neuro-Oncology. 2015;17(1):29-44.

5. Flint D, Fagan P, Panarese A. Conservative management of sporadic unilateral acoustic neuromas. The Journal of Laryngology and Otology. 2005; 19:424-428.

6. Shin YJ, Fraysse B, Cognard C, et al. Effectiveness of conservative management of acoustic neuromas. American Journal of Otolaryngology. 2000; 21:857-862

7. Day JD, Fukushima T, The surgical management of Trigeminal neuromas. Neurosurgery. 1998; 42:233-241

8. Kondziolka D, Lunsford LD, McLaughlin MR, Flickinger JC. Long-term outcome after Radiosurgery for acoustic neuroma. The New England Journal of Medicine. 1998; 339:1426-1433

9. MacNally SP, Rutherford SA, Ramsden RT, Evans DG et al. Trigeminal Schwanommas. British Journal of Neurosurgery. 2008;22(6):729-738

10. Kondziolka D, Shin SM, Brunswick A, Kim I, Silverman JS. The biology of Radiosurgery and its clinical applications for brain tumors. Neuro-Oncology. 2015;17(1):29-44.

11. Pollock BE, Lunsford LD, Kondziolka D, Flickinger JC et al. Outcome analysis of acoustic neuroma management: A comparison of microsurgery and Stereotactic Radiosurgery. Neurosurgery. 1995; $36: 215-229$

12. Pollock BE, Driscoll CL, Foote RL, et al. Patient outcomes after vestibular schwannoma management: a prospective comparison of microsurgical resection and Stereotactic Radiosurgery. Neurosurgery. 2006;59(1):77-85
13. O'Reilly BF, Mehana H, Kishore A, Crowther JA. The growth rate of non-vestibular intracranial schwannomas. Clinical Otolaryngology and Allied Science. 2004;299(1):94-97

14. Hajioff D, Rault VV, Walsh RM et al. Conservative management of Vestibular schwannomas: Third review of 10-year Prospective Study. Clincal Otolaryngology. 2008; 33:255-259.

15. Niranjan A, Barnett S, Anand V, Agazzi S. Multimodality Management of Trigeminal Schwannomas. Journal of Neurosurgical Surgery - Part B Skull Base. 2016;77(4):371-378.

16. Combs SE, Thalmann C, Debus et al. Long-term outcome of stereotactic Radiosurgery (SRS) in patients with acoustic neuromas. International Journal of Radiation Oncology Biology Physics. 2006;64(5):1341-1347

17. Hayhurst C, Monsalves E, Bernstein $M$ et al. Predicting non-auditory adverse radiation effects following Radiosurgery for vestibular schwannoma: a volume and dosimetric analysis. International Journal of Radiation Oncology Biology Physics. 2012;82(5):2041-2046

18. Murphy ES, Barnett GH, Vogelbaum MA et al. Long term outcome of Gamma Knife Radiosurgery in Patients with Vestibular Schwannomas. Journal of Neurosurgery. 2011;114(2):432-440

19. Benita H, Heyes G, Nightingale P, Hartley A, et al. Linear Accelerator Stereotactic Radiosurgery for Vestibular Schwannomas: A UK Series. Clinical Oncology. 2014;26(6):309-315

20. Mebanta SR, Buatti JM, Friedman WA, Meeks SL, Mendenhall WM, Bova FJ. Linear accelerator radiosurgery for non-acoustic schwannomas. International Journal of Radiation Oncology Biology Physics. 1999; 43:545-548

21. Pan L, Wnag EM, Zhang N, Zhou LF, Wang BJ et al. Long-term results of Lekshell gamma knife surgery for trigeminal schwannomas. Journal of Neurosurgery. 2005;102[suppl]:220-224 22. Nettel B, Niranjan A, Martin JJ, Koebbe CJ, Kondziolka D, Flickinger JC, Lunsford LD: Gamma knife radiosurgery for trigeminal schwannomas. Surgical Neurology. 2004; 62:435-446

23. Yomo S, Arkha Y, Delsanti C, Roche, PH, Thomassin JM, Jean R. Repeat Gamma Knife surgery for regrowth of vestibular schwannomas. Neurosurgery. 2009; 64:48-54

24. Flickinger JC, Kondziolka D, Niranjan A, Maitz A, Voynov G, Lunsford LD. Acoustic neuroma Radiosurgery with a marginal dose of 12-13 Gy. International Journal of Radiation Oncology Biology Physics. 2004; 60(1):225-230.

25. Hall E, Giacca A. Radiobiology for the radiologist. 6th ed. Philadelphia, PA: Lippincott Williams \& Wilkins; 2006

26. Hansasuta A, Choi CY, Gibbs IC, Soltys SG, Tse VC, Lieberson RE, Hayden MG et al. Multisession Stereotactic Radiosurgery for Vestibular Schwannomas: Single-Institution Experience With 383 Cases. Neurosurgery. 2011; 69(6):1200-1209

27. Kim KM, Park CK, Chung HT, et al. Long-term outcome of gamma knife stereotactic radiosurgery of vestibular schwannomas. Journal of Korean Neurosurgical Society 2007;42(4): 286-292

28. Erin S, John H: Radiotherapy for vestibular schwannomas: a critical review. International Journal of Radiation Oncology Biology Physics. 2011;79(4):985-997 